\title{
Ubiquitin specific peptidase 49 inhibits renal fibrosis through protein phosphatase magnesium-dependent1A- mediated Smad2/3 pathway
}

\section{Type}

Research paper

\author{
Keywords \\ renal fibrosis, EMT, USP49, PPM1A, Smad2/3
}

\begin{abstract}
Introduction

Renal fibrosis is one of the common pathologies of chronic kidney disease. This study aimed to investigate the function of ubiquitin specific peptidase 49 (USP49) in renal fibrosis and to explore the underlying mechanism
\end{abstract}

\section{Material and methods}

After analyzing the correlation between UPS49 and Smad2/3 pathways, we explored the effect of transforming growth factor- $\beta 1$ (TGF- $\beta 1$ ) on the expression of USP49. Then, the USP49 knockdown and ectopic expression human kidney-2 (HK-2) cell lines were constructed to investigate the role of USP49 in fibrosis, by determining the expression of epithelial-to-mesenchymal transition (EMT) markers (E-cadherin, $\alpha$-SMA, and vimentin), phosphorylated Smad2/3 ( $p$-Smad2/3), and protein phosphatase magnesium-dependent1A (PPMIA). Coimmunoprecipitation and ubiquitination analyses were used to determine the direct interaction between USP49 and PPM1A. The PPM1Aoverexpressed HK-2 cells were further introduced to evaluate the effects of USP49 on fibrosis. The unilateral ureteral obstruction (UUO) rats were introduced to confirm the UPS49 function in renal fibrosis in vivo.

\section{Results}

USP49 was negatively correlated with Smad2/3 pathway, and TGF- $\beta 1$ inhibited the USP49 expression. In HK-2 cells, USP49 overexpression suppressed the activity of $\alpha$-SMA and p-Smad-2/3 and activated E-cadherin, vimentin, and PPMIA, whereas USP49 knockdown displayed the reverse effects. USP49 could form a complex with PPM1A. USP49 positively regulated PPM1A expression through deubiquitination. Moreover, the fibrotic effects of USP49 knockdown were significantly attenuated with ectopic expression of PPM1A. The anti-fibrotic effect was confirmed with low expressed USP49 and PPM1A in vivo.

\section{Conclusions}

USP49 might exert anti-fibrotic effects via regulating PPM1A/Smad2/3, and USP49 might be an effective target for the treatment of renal fibrosis. 
1 Ubiquitin specific peptidase 49 inhibits renal fibrosis through protein phosphatase

4 Wenrui Liu*, Lin Liao*, Yue Guo, Jie Chen, Lianxiang Duan, Chuanfu Zhang, Jing Hu,

5 Baojuan Zhou, Jianrao $\mathrm{Lu}^{\triangle}$

6

7

8 Chinese Medicine, No. 358 Datong Street, Pudong New Area, Pudong, Shanghai 200137,

9 China

10

\section{*Contributed equally}

\section{${ }^{\triangle}$ Corresponding to:}

Jianrao Lu, Tel: +86 021-58670561, FAX: +86 021-58670561, Email: jianraolu@ 163.com.

\section{Running title: USP49 ameliorates renal fibrosis}

\section{Funding}

This work was supported by the Summit Discipline of Clinical Traditional Chinese Medicine in Pudong New Area of Shanghai (PDZY-2018-0601), and the Talents Training Program of Seventh People's Hospital of Shanghai University of TCM (XX2018-07). 


\section{Abstract}

Introduction: Renal fibrosis is one of the common pathologies of chronic kidney disease. This study aimed to investigate the function of ubiquitin specific peptidase 49 (USP49) in renal fibrosis and to explore the underlying mechanism.

Methods and materials: After analyzing the correlation between UPS49 and Smad2/3 pathways, we explored the effect of transforming growth factor- $\beta 1$ (TGF- $\beta 1$ ) on the expression of USP49. Then, the USP49 knockdown and ectopic expression human kidney-2 (HK-2) cell lines were constructed to investigate the role of USP49 in fibrosis, by determining the expression of epithelial-to-mesenchymal transition (EMT) markers (E-cadherin, $\alpha$-SMA, and vimentin), phosphorylated $\operatorname{Smad} 2 / 3 \quad(\mathrm{p}-\mathrm{S} \operatorname{mad} 2 / 3)$, and protein phosphatase magnesium-dependent1A (PPMIA). Coimmunoprecipitation and ubiquitination analyses were used to determine the direct interaction between USP49 and PPM1A. The PPM1Aoverexpressed HK-2 cells were further introduced to evaluate the effects of USP49 on fibrosis. The unilateral ureteral obstruction (UUO) rats were introduced to confirm the UPS49 function in renal fibrosis in vivo.

Results: USP49 was negatively correlated with Smad2/3 pathway, and TGF- $\beta 1$ inhibited the USP49 expression. In HK-2 cells, USP49 overexpression suppressed the activity of $\alpha$-SMA and p-Smad-2/3 and activated E-cadherin, vimentin, and PPMIA, whereas USP49 knockdown displayed the reverse effects. USP49 could form a complex with PPM1A. USP49 positively regulated PPM1A expression through deubiquitination. Moreover, the fibrotic effects of USP49 knockdown were significantly attenuated with ectopic expression of PPM1A. The anti-fibrotic effect was confirmed with low expressed USP49 and PPM1A in vivo. 
Conclusion: USP49 might exert anti-fibrotic effects via regulating PPM1A/Smad2/3, and USP49 might be an effective target for the treatment of renal fibrosis.

Keywords: Renal fibrosis, EMT, USP49, PPM1A, Smad2/3

\section{Introduction}

Chronic kidney disease (CKD) could cause loss of healthy renal structure and contribute to end-stage renal disease associated with excessive deposition of extracellular matrix (ECM)[1]. Renal fibrosis is considered the final common pathological feature of most CKD, including diabetic nephropathy, renal vascular dysfunction, glomerular hypertension, increased susceptibility, and, eventually, loss of tubular cells [2-5]. Fibrosis is characterized by the excessive ECM molecules, primarily collagens produced by the ECM-producing cells such as fibroblasts and their activated counterparts, myofibroblasts [6, 7].

The actions of fibroblasts are differentiated into myofibroblasts, and epithelial-to-mesenchymal transition (EMT) is regulated by many factors, including cytokines, ECM components, and mechanical stress $[8,9]$. Besides, the reports showed that multiple signaling pathways are also activated during renal fibrosis, including TGF- $\beta /$ Smads, Wnt/B-catenin, c-Jun N-terminal kinase (JNK)/STAT3, and mitogen-activated protein kinase (MAPK) [10-13]. Increasing evidence has shown that the TGF- $\beta /$ Smads signaling pathway located the major driver. The activated TGF- $\beta /$ Smad pathway has been found in different cell types from various renal disease models [14]. Briefly, TGF- $\beta 1$ binds to the TGF- $\beta$ receptor, and Smad direct and indirect pathways were activated. Then, the $\mathrm{p}-\mathrm{Smad} 2 / 3$ complex translocates into the nucleus, and target genes will be transcribed. This leads to ECM synthesis stimulation, degradation suppression, and tubular epithelial cells and endothelial 
cells transcribed to EMT or endothelial-mesenchymal transition (EndoMT) [15-18].

PPMIA is the Ser/Thr protein phosphatase and has been reported to be involved in several signaling pathways, such as p38, JNK, Wnt, and p53 [19-22]. It is identified that PPM1A is the only phosphatase for Smad2 and Smad3, dephosphorylating Smad2/3, leading to TGF- $\beta /$ Smad signaling blockage. PPM1 A has been reported to be involved in the liver and kidney fibrosis [23-25]. And the report has shown that PPM1A was deubiquitinated by ubiquitin specific peptidase 33(USP33) in lung cancer [26].

USP49, another member of the USP family, is reported in the regulation of pre-mRNA splicing, suppressing tumorigenesis in pancreatic cancer by targeting FKBP5-protein kinase B (Akt) signaling and inhibiting non-small-cell lung cancer by targeting phosphatidylinositol 3-kinase (PI3K)/Akt pathway[27, 28]. However, no known direct role of USP49 has been revealed in renal fibrosis.

In the current study, we found that the USP49 was negatively correlated with Smad2/3, suppressed by TGF- $\beta 1$. USP49 significantly inhibited renal fibrosis in HK-2 cells. Further investigation revealed that the anti-fibrotic effects of USP49 were mainly through inhibiting the TGF- $\beta /$ Smad2/3 pathway, and PPM1A was required for this. To the best of our knowledge, this research presents the first evidence and mechanism of USP49 in renal fibrosis.

\section{Material and methods}

Data source and functional enrichment analysis

The microarray data of GSE7392 were downloaded from the National Center for Biotechnology Information Gene Expression Omnibus (GEO) database (https://www.ncbi.nlm.nih.gov/geo/query/acc.cgi?acc=GSE7392) with samples from 16 
fibrosis patients and 14 healthy controls. The functional enrichment analysis was implemented using Gene Set Enrichment Analysis version 3.0 (GSEA, https://www.genome.jp/kegg/) with the adjusted $p$ value $<0.05$.

Plasmids

The USP49 (AJ586139.1) gene was cloned into pLVX-Puro vector (Clontech) using EcoRI and BamHI with primers (Table 1).

The PPMIA gene was cloned into pLVX-Puro vector (Clontech) using EcoRI and BamHI with primers (Table 1).

Cell culture and transfection

293 T cells were purchased from American Type Culture Collection (ATCC, VA, USA) and were cultured with DMEM (Gibco, CA, USA) supplemented with 10\% FBS. Cells were cultured at $37^{\circ} \mathrm{C}$ under a humidified $5 \% \mathrm{CO}_{2}$.

The pLVX-Puro-USP49, psPAX2, and pMD2G (Addgene) were cotransfected into 293T cells using Lipofectamine ${ }^{\mathrm{TM}} 2000$ (Invitrogen, CA, USA). Then, cells were cultured in a complete medium after 6-hour incubation, and lentiviruses were harvested at 48 hours and 72 hours. HK-2 cells were transfected with $1.5 \mu \mathrm{g}$ of pLVX-Puro-USP49 using Lipofectamine 2000 reagent. Cells transfected with pLVX-Puro were used as the control.

Stable cell line construction

Three different shRNAs were synthesized and inserted into pLKO.1 vectors (pLKO.1-shUSP49) using primers (Table 1). The plasmids were confirmed by Shanghai Majorbio Bio-Pharm Technology Co., Ltd. 293T cells were cotransfected with pLKO.1-shUSP49, psPAX2, and pMD2G (Addgene). The scrambled shRNA served as 
111

control. After incubation for $72 \mathrm{~h}$, the virus was harvested. HK-2 cells were infected with a virus to develop the USP49 knockdown stable cell line.

Western blotting

Total protein concentration was determined by BCA protein assay kit according to the manufacturer's instructions (Thermo, MA, USA). Samples were heated at $95^{\circ} \mathrm{C}$ for $10 \mathrm{~min}$, and $30 \mathrm{mg}$ of them was separated by $10 \%$ SDS-PAGE. Then, samples were transferred to PVDF membranes and blocked in 5\% skim milk for $1 \mathrm{~h}$ at room temperature(RT). The membrane was incubated with the primary antibody USP49 (1:1000, ab127574, Abcam, UK), PPM1A (1:1000, ab14824, Abcam), vimentin (1:500, ab8978, Abcam), $\alpha-S M A ~(1: 1000$, ab124964, Abcam), E-cadherin (1:500, ab1416, Abcam), Smad2/3 (1:1000, ab202445, Abcam), p-Smad2/3 (1:500, ab63399, Abcam), TGF- $\beta 1$ (1:1000, ab179695, Abcam), and GAPDH (1:2000, \#5174, CST, MA, USA) at $4^{\circ} \mathrm{C}$ overnight. Then, the membrane was incubated with secondary antibodies (A0208, A0181, and A0216, GE Healthcare/Amersham Biosciences, Piscataway, NJ, China) at RT for 1h. LAS-400 image analyzer (FujiFilm Medical Systems, CT, USA) was used to detect the HRP (GE Healthcare/Amersham Biosciences) signal.

\section{RT-PCR}

Total RNA was extracted using Trizol reagent (1596-026, Invitrogen, CA, USA). cDNA library was constructed using Revert Aid First Strand cDNA Synthesis Kit (\#K1622, Fermentas, CA, USA) according to the manufacturer's instructions. SYBR Green PCR Mix (Thermo) and primers (shown in Table 2) were used to evaluate the mRNA expression of USP49, PPM1A, and GAPDH on ABI Prism 7300 SDS system (Applied Biosystem, CA, 
USA).

In vitro coimmunoprecipitation (Co-IP) and ubiquitination assay

The association between USP49 and PPM1A in HK-2 cells was assessed using Co-IP. Briefly, Protein A/G PLUS-Agarose (sc-2003, Santa Cruz, CA, USA) was used to obtain the total protein $(100 \mu \mathrm{g})$ from cell lysis supernatant. IgG (sc-2027, Santa Cruz), anti-USP49 antibody (NBP1-81173, NOVUS, CT, USA), and antibody against PPM1A (NBP1-04333, NOVUS) were used for IP. Anti-USP49 antibody (ab127574, Abcam) and anti-PPM1A antibody (ab14824, Abcam) were used for Westernblot.An anti-ubiquitin antibody (ab7780, Abcam) was used to determine the PPM1A ubiquitination (Ub-PPM1A).

Unilateral ureteral obstruction (UUO) model construction

Six-week-old male SD rats $(160 \pm 20 \mathrm{~g})$ were obtained from the Shanghai Laboratory Animal Center (Shanghai, China). All rats were kept in a temperature-controlled house $\left(25 \pm 1{ }^{\circ} \mathrm{C}\right)$ with free access to food and water. Twelve SD rats were randomly divided into two groups: the control group and the renal fibrosis group.

Renal fibrosis was induced by UUO according to a previous study [29].

Histology evaluation

On the 4th and 8th weeks, the renal tissues were collected and embedded in paraffin. Slides of $4 \mu \mathrm{m}$ thickness were sectioned. Hematoxylin-eosin (H\&E) staining was performed to observe the histological changes, and Masson's trichrome staining was carried out to measure the density of collagen fibers. Histology evaluation and Masson's trichrome staining were carried out and observed using an optical microscope (Olympus, Japan). For each rat, three tissue fields were examined. The reagents used in this part were as follows: hematoxylin 
155

(714094, BASO, Guangdong, China), eosin (BA4099, BASO), and Masson's staining (Leagene Biotechnology Co., Ltd., Beijing, China).

Urea measurements

Colorimetric assay (Diasys Diagnostic System,Holzheim, Germany) was used to determine the urea concentration according to the manufacturer's instructions. The baseline was generated using standard urea (Diasys Diagnostic Systems).

Statistical analyses

Each experiment was independently repeated three times. The data were shownas the mean \pm standard error of the mean. Student's $t$-test was used between two groups. One-way analysis of variance with post hoc Tukey's test was used between multiple groups. $p<0.05$ was regarded as statistically significant.

\section{Results}

USP49 negatively correlated with Smad2/3 pathway

To explore the gene expression profile in renal fibrosis progression, the microarray data of the expression profile of GSE7392 wereobtained including 16 fibrosis and 14 healthy controls. We found a lower expression of USP49 in renal fibrotic samples compared with the control samples (Figure1A). As Smad2/3 pathways dominated the renal fibrosis transition, we identified the correlation between USP49 and Smad2/3 pathway. Functional analysis revealed the negative correlation between USP49 and Smad2/3 (Figure1B). These results indicated thatUSP49 might function in renal fibrosis.

USP49 was suppressed by TGF- $\beta 1$

The TGF- $\beta 1 / \mathrm{Smad} 2 / 3$ signaling pathway plays a central role in renal fibrosis, and 
TGF- $\beta 1$ initialized this pathway by the phosphorylation and activation of Smad2/3. To explore the TGF- $\beta 1$ effect on USP49, we investigated the expression of USP49 by stimulation of different concentrations of TGF- $\beta 1$. We found that TGF- $\beta 1$ inhibited the mRNA and protein expression of USP49 in HK-2 cells in a dose-dependent manner (Figure 2).

USP49 inhibited the expression of EMT-related proteins

Next, to confirm the effect of USP49 on renal fibrosis, USP49 stable knockdown with three separate shRNAs and stable ectopic expression HK-2 human renal epithelial cell lines were constructed. Compared with the control group, over 80\% decrease in USP49 protein expression was observed, and we chose the most significant knockdown cell lines for further analysis (Figure 3A). We observed that TGF- $\beta 1$ could induce EMT, with a significant decrease in E-cadherin expression and a remarkable increase in the vimentin and $\alpha$-SMA expression. P-Smad2/3 was also significantly increased after TGF- $\beta 1$ induction, which showed successfully TGF- $\beta 1 / \operatorname{Smad} 2 / 3$ pathway activation in EMT. PPM1A, the only phosphatase for Smad2/3 in EMT, was found to be significantly decreased, but USP49 overexpression reversed this phenotype (Figure 3B). To further validate the renal fibrosis dedifferentiation effect of USP49, we explored the protein expression in the EMT process. Consistent with our previous thought, UPS49 knockdown significantly promoted the EMT process. PPM1A and E-cadherin were significantly suppressed, while vimentin, $\alpha$-SMA, and p-Smad $2 / 3$ were significantly increased (Figure 3C). These results supported our hypothesis that USP49 indeed functioned in EMT.

USP49 directly interacted with PPM1A PPM1A caught our attention as there was positive correlation between USP49 and 
199

PPM1A. PPM1A was also regulated by deubiquitination. We proposed that USP49 played a role in renal EMT through PPM1A. We found that several results supported our hypothesis. Firstly, we foundthatUSP49 regulated the expression of PPM1A. The protein expression of PPM1A was increased in USP49overexpressed cells, and downregulation of PPM1A was revealed in USP49 knockdown cells, although there was no effect on the mRNA profile of PPM1A (Figure4A). We next explored the potential interaction between USP49 and PPM1A. Co-IP assays demonstrated that UAP49 formed a complex with PPM1A (Figure4B). To determine whether USP49 could directly deubiquitinate PPM1A, we performed the in vitro deubiquitination assay. We found that USP49 overexpression could dramatically deubiquitinatePPM1A in vitro (Figure4C). To further confirm our data, proteasome inhibitor MG132 was added toUSP49 stable knockdown cells. We found that MG132 significantly inhibited the degradation of PPM1A (Figure4D). These results were consistent with our proposal that USP49 bound directly toPPM1A and protected it from degradation by deubiquitination.

USP49 functioned in EMT through PPM1A

To further confirm our previous thought, PPM1A overexpressed cell line was developed with significantly upregulated USP49 (Figure5A). USP49 knockdown could remarkably promote renal EMT with a significant decrease in E-cadherin and PPM1A and a remarkable increase in vimentin, $\alpha$-SMA, and p-Smad2/3, while this effect was attenuated by PPM1A (Figure5B). Taking all the above results together, USP49 might bind toPPM1A and prevent it from degradation and function in EMT dedifferentiation.

In vivo function of USP49 in renal fibrosis 

reproducible model. To identify the anti-EMT effect of USP49, the UUO rat was developed.

223 On the 4th and 8th weeks, HE staining and Masson's trichrome staining were performed to evaluate the fibrotic phenotype. Moreover, creatinine, urea nitrogen in serum, and urinary protein in urea were elevated in the UUO group compared with the control group on weeks 4 and 8. These results confirmed the successful UUO model development. Next, we explored EMT-related protein expression and USP49. Consistent with our previous hypothesis, there was a significant decrease in USP49, E-cadherin, and PPM1A and a remarkable increase in the expression of vimentin, $\alpha$-SMA, and $\mathrm{p}-\mathrm{Smad} 2 / 3$ in the UUO group compared with the control group on both the 4th and 8th weeks.

\section{Discussion} due to limited effective treatments, and it is often accompanied by the occurrence of other has been reported to function in pre-RNA splicing during tumorigenesis [28, 34-36]. Herein, progress and activate the PPM1A. Further investigation revealed the direction between we found that USP49 was negatively correlated withtheSmad2/3 pathway and inhibited by TGF- $\beta 1$. To investigate the function in renal fibrosis, USP49 knockdown and overexpressed HK-2 stable cell lines were constructed. We found that USP49 could suppress the EMT USP49 and PPM1A, and the PPM1A was stabilized through deubiquitination by USP49. 
Moreover, PPM1A reversed the EMT effect by USP49 knockdown. At last, we further confirmed the anti-fibrotic effect of USP49 on the rat UUO model in vivo. To the best of our knowledge, our finding firstly elucidates the anti-fibrotic function of USP9 and indicates the clinical potential in CKD.

Several mechanisms have been explored in renal fibrosis. ECM synthesis is induced by TGF- $\beta 1$ via Smad3-dependent or Smad3-independent manners. Matrix metalloproteinase (MMP) suppresses the degradation of ECM. TGF- $\beta 1 / \mathrm{Smad} 2 / 3$ plays critical roles in transdifferentiation toward myofibroblasts from several cell types such as epithelial cells via EMT, EndoMT, and pericytes and bone marrow-derived macrophages via macrophage-myofibroblast transition (MMT) [1, 37]. In our study, we found that USP49 was negatively correlated with Smad2/3 and inhibited by TGF- $\beta 1$. EMT biomarkers illustrated that the TGF- $\beta 1$ could inactivate the EMT transition and played roles in renal fibrosis.

Besides the canonical TGF- $\beta 1 / \mathrm{Smad} 2 / 3$ pathway, noncanonical pathways were also correlated withtheSmad2/3 pathway, such as extracellular signal-regulated kinase (ERK),

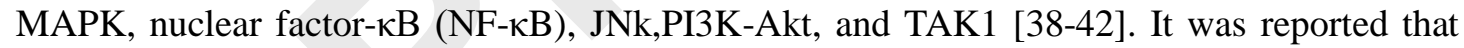
USP49 inhibited non-small-cell lung cancer cell growth by PI3K/Akt pathway [43]. USP49 also regulated the DUSP1-JNK1/2 pathway [36]. These data indicated that USP49 might also play roles through the noncanonical pathway.

As a serine/threonine phosphatase, PPM1A regulates bone morphogenetic protein and TGF- $\beta$ signaling pathways by dephosphorylating its substrates such as MAPK and Smad1/2/3 [44]. Enhanced PPM1A efficiently blocked the human hepatic fibrosis [23]. Downregulated PPM1A has been observed in HBV virus protein-related HCC [45]. Ectopic PPM1A 
expression could reverse Smad2/3 and mediate kidney fibrotic gene induction [24]. Our data showed that the USP49 bound directly toPPM1A, and a positive correlation between USP49 and PPM1A was observed using USP49 knockdown and overexpressed cell lines. Moreover, PPM1A could reverse the EMT in USP49 knockdown cells. These results indicated that the anti-fibrotic effect of USP49 was mediated by PPM1A.

E3 ubiquitin ligase could promote proteasome-mediated protein degradation, which functions in many cell processes such as inflammation, cell growth, proliferation, apoptosis, and survival. Increasing evidence showed that the reversal of ubiquitination modification plays essential roles in various physiological processes [46]. USP49 is a deubiquitinase and plays roles in biological functions. USP49 negatively regulated tumorigenesis and chemoresistance throughtheFKBP51-Akt signaling pathway [35]. USP49 could also serve as the novel tumor suppressor in tumors at least in NSCLC and pancreatic cancer. Forming a positive feedback loop with p53, USP49 could also participate in the DNA damage response [28]. Our study revealed that PPM1A could be deubiquitinated by USP49, and thus EMT could be reversed. Furthermore, consistent with our proposal, the anti-fibrotic effect was confirmed in the UUO model in vivo.

\section{Conclusion}

Our study indicated that USP49 might exert anti-fibrotic effects via regulating PPM1A/Smad2/3 by direct interaction with PPM1A and stabilizing PPM1A through deubiquitination. This suggests that USP49 may be a novel target for renal fibrosis. On the other hand, there are some questions that remained to be further explored: the USP49isregulated by TGF- $\beta 1$ and the function needs to be investigated; the direct interaction 
needs to be revealed between USP49 and Smad2/3; the mechanism of USP49 anti-fibrotic effect should be confirmed in vivo.

\section{References}

[1] Meng XM, Nikolic-Paterson DJ and Lan HY. TGF- $\beta$ : the master regulator of fibrosis. Nat Rev Nephrol 2016; 12: 325-338.

[2] Bader R, Bader H, Grund KE, Mackensen-Haen S, Christ H and Bohle A. Structure and function of the kidney in diabetic glomerulosclerosis. Correlations between morphological and functional parameters. Pathol Res Pract 1980; 167: 204-216.

[3] García-Sánchez O, López-Hernández FJ and López-Novoa JM. An integrative view on the role of TGF-beta in the progressive tubular deletion associated with chronic kidney disease. Kidney Int 2010; 77: 950-955.

[4] Grande MT, Pérez-Barriocanal F and López-Novoa JM. Role of inflammation in túbulo-interstitial damage associated to obstructive nephropathy. J Inflamm (Lond) 2010; 7: 19.

[5] Robak E, Gerlicz-Kowalczuk Z, Dziankowska-Bartkowiak B, Wozniacka A and Bogaczewicz J. Serum concentrations of IL-17A, IL-17B, IL-17E and IL-17F in patients with systemic sclerosis. Arch Med Sci 2019; 15: 706-712.

[6] Strutz F and Zeisberg M. Renal fibroblasts and myofibroblasts in chronic kidney disease. J Am Soc Nephrol 2006; 17: 2992-2998.

[7] Grande MT and López-Novoa JM. Fibroblast activation and myofibroblast generation in obstructive nephropathy. Nat Rev Nephrol 2009; 5: 319-328. 
[8] Darby IA and Hewitson TD. Fibroblast differentiation in wound healing and fibrosis. Int Rev Cytol 2007; 257: 143-179.

[9] Zheng P, Li H, Xu P, Wang X, Shi Z, Han Q and Li Z. High lncRNA HULC expression is associated with poor prognosis and promotes tumor progression by regulating epithelial-mesenchymal transition in prostate cancer. Arch Med Sci 2018; 14: 679-686.

[10] Furukawa F, Matsuzaki K, Mori S, Tahashi Y, Yoshida K, Sugano Y, Yamagata H, Matsushita M, Seki T, Inagaki Y, Nishizawa M, Fujisawa J and Inoue K. p38 MAPK mediates fibrogenic signal through Smad3 phosphorylation in rat myofibroblasts. Hepatology 2003; 38: 879-889.

[11] Yu X, Lin SG, Huang XR, Bacher M, Leng L, Bucala R and Lan HY. Macrophage migration inhibitory factor induces MMP-9 expression in macrophages via the MEK-ERK MAP kinase pathway. J Interferon Cytokine Res 2007; 27: 103-109.

[12] Lim AK, Nikolic-Paterson DJ, Ma FY, Ozols E, Thomas MC, Flavell RA, Davis RJ and Tesch GH. Role of MKK3-p38 MAPK signalling in the development of type 2 diabetes and renal injury in obese db/db mice. Diabetologia 2009; 52: 347-358.

[13] Sun YB, Qu X, Li X, Nikolic-Paterson DJ and Li J. Endothelial dysfunction exacerbates renal interstitial fibrosis through enhancing fibroblast Smad3 linker phosphorylation in the mouse obstructed kidney. PLoS One 2013; 8: e84063.

[14] Huang XR, Chung AC, Wang XJ, Lai KN and Lan HY. Mice overexpressing latent TGF-beta1 are protected against renal fibrosis in obstructive kidney disease. Am J Physiol Renal Physiol 2008; 295: F118-127.

[15] Li J, Qu X, Yao J, Caruana G, Ricardo SD, Yamamoto Y, Yamamoto H and Bertram JF. Blockade of endothelial-mesenchymal transition by a Smad3 inhibitor delays the early 
[16] Mariasegaram M, Tesch GH, Verhardt S, Hurst L, Lan HY and Nikolic-Paterson DJ. Lefty antagonises TGF-beta1 induced epithelial-mesenchymal transition in tubular epithelial cells. Biochem Biophys Res Commun 2010; 393: 855-859.

[17] Qin W, Chung AC, Huang XR, Meng XM, Hui DS, Yu CM, Sung JJ and Lan HY. TGF- $\beta$ /Smad3 signaling promotes renal fibrosis by inhibiting miR-29. J Am Soc Nephrol $2011 ; 22: 1462-1474$.

[18] Loeffler I and Wolf G. Epithelial-to-Mesenchymal Transition in Diabetic Nephropathy: Fact or Fiction? Cells 2015; 4: 631-652.

[19] Takekawa M, Maeda T and Saito H. Protein phosphatase 2Calpha inhibits the human stress-responsive p38 and JNK MAPK pathways. Embo j 1998; 17: 4744-4752.

[20] Strovel ET, Wu D and Sussman DJ. Protein phosphatase 2Calpha dephosphorylates axin and activates LEF-1-dependent transcription. J Biol Chem 2000; 275: 2399-2403.

[21] Zolnierowicz S. Type 2A protein phosphatase, the complex regulator of numerous signaling pathways. Biochem Pharmacol 2000; 60: 1225-1235.

[22] Ofek P, Ben-Meir D, Kariv-Inbal Z, Oren M and Lavi S. Cell cycle regulation and p53 activation by protein phosphatase 2C alpha. J Biol Chem 2003; 278: 14299-14305.

[23] Wang L, Wang X, Chen J, Yang Z, Yu L, Hu L and Shen X. Activation of protein serine/threonine phosphatase PP2C $\alpha$ efficiently prevents liver fibrosis. PLoS One 2010; 5: e14230.

[24] Samarakoon R, Rehfuss A, Khakoo NS, Falke LL, Dobberfuhl AD, Helo S, Overstreet JM, Goldschmeding $\mathrm{R}$ and Higgins PJ. Loss of expression of protein phosphatase 
magnesium-dependent $1 \mathrm{~A}$ during kidney injury promotes fibrotic maladaptive repair. Faseb $\mathrm{j}$ 2016; 30: 3308-3320.

[25] Zhou J, Lan Q, Li W, Yang L, You J, Zhang YM and Ni W. Tripartite motif protein 52 (TRIM52) promoted fibrosis in LX-2 cells through PPM1A-mediated Smad2/3 pathway. Cell Biol Int 2019;

[26] Lu J, Zhong Y, Chen J, Lin X, Lin Z, Wang N and Lin S. Radiation Enhances the EpithelialMesenchymal Transition of A549 Cells via miR3591-5p/USP33/PPM1A. Cell Physiol Biochem 2018; 50: 721-733.

[27] Sun Y, Liu WZ, Liu T, Feng X, Yang N and Zhou HF. Signaling pathway of MAPK/ERK in cell proliferation, differentiation, migration, senescence and apoptosis. J Recept Signal Transduct Res 2015; 35: 600-604.

[28] Tu R, Kang W, Yang X, Zhang Q, Xie X, Liu W, Zhang J and Zhang XD. USP49 participates in the DNA damage response by forming a positive feedback loop with p53. Cell Death Dis 2018; 9: 553.

[29] Song J, Liu J, Luo J, Zhang Q, Xia Y, Shao Q, Sun C, Jiang C, Zhang M and Zhu W. A modified relief of unilateral ureteral obstruction model. Ren Fail 2019; 41: 497-506.

[30] Sevencan NO and Ozkan AE. Associations between neutrophil/lymphocyte ratio, platelet/lymphocyte ratio, albuminuria and uric acid and the estimated glomerular filtration rate in hypertensive patients with chronic kidney disease stages 1-3. Arch Med Sci 2019; 15: 1232-1239.

[31] Kadri AN, Kaw R, Al-Khadra Y, Abuamsha H, Ravakhah K, Hernandez AV and Tang WHW. The role of B-type natriuretic peptide in diagnosing acute decompensated heart failure in 
chronic kidney disease patients. Arch Med Sci 2018; 14: 1003-1009.

[32] Marcucci F, Stassi G and De Maria R. Epithelial-mesenchymal transition: a new target in anticancer drug discovery. Nat Rev Drug Discov 2016; 15: 311-325.

[33] Yeung KT and Yang J. Epithelial-mesenchymal transition in tumor metastasis. Mol Oncol 2017; 11: 28-39.

Zhang Z, Jones A, Joo HY, Zhou D, Cao Y, Chen S, Erdjument-Bromage H, Renfrow M, He H, Tempst P, Townes TM, Giles KE, Ma L and Wang H. USP49 deubiquitinates histone H2B and regulates cotranscriptional pre-mRNA splicing. Genes Dev 2013; 27: 1581-1595.

[35] Luo K, Li Y, Yin Y, Li L, Wu C, Chen Y, Nowsheen S, Hu Q, Zhang L and Lou Z. USP49 negatively regulates tumorigenesis and chemoresistance through FKBP51-AKT signaling. Embo j 2017; 36: 1434-1446.

[36] Zhang W, Zhang Y, Zhang H, Zhao Q, Liu Z and Xu Y. USP49 inhibits ischemia-reperfusion-induced cell viability suppression and apoptosis in human AC16 cardiomyocytes through DUSP1-JNK1/2 signaling. J Cell Physiol 2019; 234: 6529-6538.

[37] Wu CF, Chiang WC, Lai CF, Chang FC, Chen YT, Chou YH, Wu TH, Linn GR, Ling H, Wu KD, Tsai TJ, Chen YM, Duffield JS and Lin SL. Transforming growth factor $\beta$-1 stimulates profibrotic epithelial signaling to activate pericyte-myofibroblast transition in obstructive kidney fibrosis. Am J Pathol 2013; 182: 118-131.

[38] Derynck R and Zhang YE. Smad-dependent and Smad-independent pathways in TGF-beta family signalling. Nature 2003; 425: 577-584.

[39] Hayashida T, Decaestecker M and Schnaper HW. Cross-talk between ERK MAP kinase and Smad signaling pathways enhances TGF-beta-dependent responses in human mesangial cells. 
[40] Ono K, Ohtomo T, Ninomiya-Tsuji J and Tsuchiya M. A dominant negative TAK1 inhibits cellular fibrotic responses induced by TGF-beta. Biochem Biophys Res Commun 2003; 307: $332-337$.

[41] Xie L, Law BK, Chytil AM, Brown KA, Aakre ME and Moses HL. Activation of the Erk

[43] Shen WM, Yin JN, Xu RJ, Xu DF and Zheng SY. Ubiquitin specific peptidase 49 inhibits non-small cell lung cancer cell growth by suppressing PI3K/AKT signaling. Kaohsiung J Med Sci 2019; 35: 401-407.

[44] Lin X, Duan X, Liang YY, Su Y, Wrighton KH, Long J, Hu M, Davis CM, Wang J, Brunicardi FC, Shi Y, Chen YG, Meng A and Feng XH. PPM1A functions as a Smad phosphatase to terminate TGFbeta signaling. Cell 2006; 125: 915-928.

[45] Liu Y, Xu Y, Ma H, Wang B, Xu L, Zhang H, Song X, Gao L, Liang X and Ma C. Hepatitis B virus X protein amplifies TGF- $\beta$ promotion on HCC motility through down-regulating PPM1a. Oncotarget 2016; 7: 33125-33135.

[46] Mevissen TET and Komander D. Mechanisms of Deubiquitinase Specificity and Regulation. Annu Rev Biochem 2017; 86: 159-192. 


\section{Figure legends}

Figure 1. Clinical significance of USP49 in human renal fibrosis. (A) The correlation analysis between USP49 and renal fibrosis was retrieved from the GEO database. The expression of USP49 in the healthy group and renal fibrosis group was calculated. GAPDH was used as the internal control. ${ }^{*} p=0.02$. (B) Running enrichment score was negative between USP49 and Smad2/3 pathways.

Figure 2. Recombinant TGF- $\beta 1$ protein inhibited USP49 expression. HK-2 cells were induced by different concentrations of TGF- $\beta 1$ (ng/mL). USP49 mRNA was measured using qRT-PCR (Left panel); the protein expression of USP49 was measured by Westernblot (right panel). GAPDH severed as the loading control. Bar indicated the expression related to $0 \mathrm{ng} / \mathrm{mL}$ group. Data were expressed as mean $\pm \mathrm{SD} .{ }^{* *} p<0.01$.

Figure 3. Roles of USP49 in HK-2 fibrogenesis and the underlying mechanism.(A) USP49 knockdown stable cell line and overexpression cell line construction. The mRNA expression of USP49 was determined by qRT-PCR (left panel). The protein expression of USP49 was determined by Western blot (right panel). GAPDH served as the loading control. (B) USP49 inhibited EMT, Smad2/3, and PPM1A. USP49 overexpressed (oeUSP49) and control HK-2 cells were treated with recombinant TGF- $\beta 1$.The USP49, PPM1A, vimentin, $\alpha$-SMA, E-cadherin, Smad2/3, and p-Smad2/3 were assessed by Westernblot. GAPDH-1 was used to normalize USP49, PPM1A, and vimentin; GAPDH-2 was used to normalize $\alpha$-SMA, E-cadherin, Smad2/3, and p-Smad2/3. (C) USP49 knockdown promoted EMT, Smad2/3 pathway activation, and PPM1A downregulation. The USP49, PPM1A, vimentin, $\alpha$-SMA, E-cadherin, Smad2/3, and p-Smad2/3 were assessed by Western blot. GAPDH-1 was used to 
normalize USP49, PPM1A, and vimentin; GAPDH-2 was used to normalize $\alpha$-SMA,

E-cadherin, $\operatorname{Smad} 2 / 3$, and $\mathrm{p}-\mathrm{Smad} 2 / 3$. Data were expressed as mean $\pm \mathrm{SD} .{ }^{* *} p<0.01$ vs. vector; ${ }^{\# \#} p<0.01$ vs. siNC; ${ }^{\Delta \Delta} p<0.01$ vs. vector+ vehicle; ${ }^{++} p<0.01$ vs. vector+ TGF- $\beta 1$.

Figure 4. USP49 bound directly toPPM1A and inhibited PPM1A ubiquitination. (A) The effect of USP49 on the expression of PPM1A. The mRNA expression of PPM1A was measured by qRT-PCR (left panel), and the protein expression of PPM1A was accessed by Western blot in USP49overexpressed HK-2 cells and knockdown HK-2 cells. GAPDH was used as loading control. (B) PPM1A was immune precipitated and immune blotted with the indicated antibody. (C) Deubiquitination of PPM1A by USP49 overexpression. HK-2 cells stably expressing USP49 were harvested for immune precipitated with PPM1A antibody and immune blotted with ubiquitin antibody. (D)USP49 stably knockdown cells were treated with MG132 for 4h before harvest. PPM1 A expression was determined by Western blot. Data were expressed as mean $\pm \mathrm{SD} .{ }^{* *} p<0.01$ vs. vector; ${ }^{\# \#} p<0.01$ vs. siNC; ${ }^{++} p<0.01$ vs. $\operatorname{sinC}+$ MG132.

Figure 5.The promoting effect of siUSP49 on HK-2 fibrogenesis was reversed by PPM1A overexpression. (A) PPM1A overexpressed stable cell line was constructed. The mRNA was determined by qRT-PCR (left panel), and the protein was measured by Western blot (right panel). GAPDH was used as loading control. (B) ThePPM1A, vimentin, $\alpha$-SMA, E-cadherin,

$458 \mathrm{Smad} / 3$, and $\mathrm{p}-\mathrm{Smad} 2 / 3$ were assessed by Western blot in USP49 knockdown, PPM1A overexpression, and USP49 knockdown in PPM1A overexpression cells. GAPDH-1 was used to normalize $\alpha$-SMA, E-cadherin, PPM1A, and vimentin; GAPDH-2 was used to normalize 
vs. $\operatorname{siNC} ;{ }^{++} p<0.01$ vs. siNC + PPM1A.

463 Figure 6. Expression of USP49 and PPM1A in rat renal fibrosis induced by UUO. HE staining

464 (A) and Masson's trichrome sating (B) were accessed to determine the renal fibrosis of UUO

465 rat on the 4thand 8thweeks. (C) Creatinine and urea nitrogen in serum and urinary protein in

466 urea were accessed by ELISA to determine the renal fibrosis of UUO rat on the 4th and 8th

467 weeks. (D) TheTGF- $\beta 1$, USP49, PPM1A, vimentin, $\alpha-S M A$, E-cadherin, Smad2/3, and

468 p-Smad2/3 were assessed by Western blot on the 4thand 8thweeks. GAPDH-1 was used to

469 normalize TGF- $\beta 1$, USP49, PPM1A, $\alpha$-SMA, E-cadherin, and vimentin in UUO rat;

470 GAPDH-2 was used to normalize $\operatorname{Smad} 2 / 3$ and $\mathrm{p}-\mathrm{Smad} / 3$. Data were expressed as mean \pm

471 SD. ${ }^{* *} p<0.01$ vs. control.

472

473 
Table 1 Primers used for the construction of lentivirus vector

\begin{tabular}{|c|c|}
\hline Description & Sequences or primers $\left(5^{\prime}-3^{\prime}\right)$ \\
\hline \multicolumn{2}{|l|}{ USP49 (AJ586139.1) } \\
\hline shRNA group 1 & CCACGCCCTGAAACACTTT \\
\hline \multirow{2}{*}{ Forward } & CCGGTCCACGCCCTGAAACACTTTCTCGAGAA \\
\hline & AGTGTTTCAGGGCGTGGTTTTTG \\
\hline \multirow{2}{*}{ Reversed } & AATTCAAAAACCACGCCCTGAAACACTTTCTC \\
\hline & GAGAAAGTGTTTCAGGGCGTGGA \\
\hline shRNA group 2 & CCGAGTTCAAAGCACATTT \\
\hline \multirow{2}{*}{ Forward } & CCGGTCCGAGTTCAAAGCACATTTCTCGAGAA \\
\hline & ATGTGCTTTGAACTCGGTTTTTG \\
\hline \multirow{2}{*}{ Reversed } & AATTCAAAAACCGAGTTCAAAGCACATTTCTC \\
\hline & GAGAAATGTGCTTTGAACTCGGA \\
\hline shRNA group 3 & GCTCACCAAACAGGTCTTA \\
\hline \multirow{2}{*}{ Forward } & CCGGTGCTCACCAAACAGGTCTTACTCGAGGC \\
\hline & TCACCAAACAGGTCTTATTTTTG \\
\hline \multirow{2}{*}{ Reversed } & AATTCAAAAAGCTCACCAAACAGGTCTTACTC \\
\hline & GAGGCTCACCAAACAGGTCTTAA \\
\hline USP49 over-expression & (AJ586139.1) CDS 1-2067 \\
\hline Forward & CGGAATTCATGGATAGATGCAAACATGTAGG \\
\hline Reversed & CGGGATCCTCAGGAAAATGTCTGTGGTCTG \\
\hline PPM1A over-expression & (NM_021003.4) CDS: 451-1599 \\
\hline Forward & CGGAATTCATGGGAGCATTTTTAGACAAGC \\
\hline Reversed & CGGGATCC CCACATATCATCTGTTGATGTAG \\
\hline
\end{tabular}


Table 2 Primers used for qRT-PCR

\begin{tabular}{llc}
\hline Gene & Primers $\left(5^{\prime}-3^{\prime}\right)$ & Description \\
\hline USP49 & TCCCACAAAGGAAGTAACC & Forward \\
& TATGACAGCAGCAAGTAGG & Reversed \\
PPM1A & CCCTTGTTTCCTCTACTTTC & Forward \\
& TAATCCTTCCCTACCTATCC & Reversed \\
GAPDH & AATCCCATCACCATCTTC & Forward \\
& AGGCTGTTGTCATACTTC & Reversed \\
\hline
\end{tabular}


A

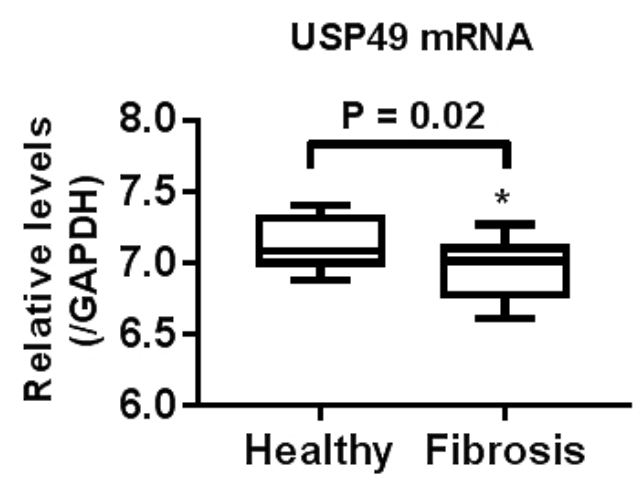

B

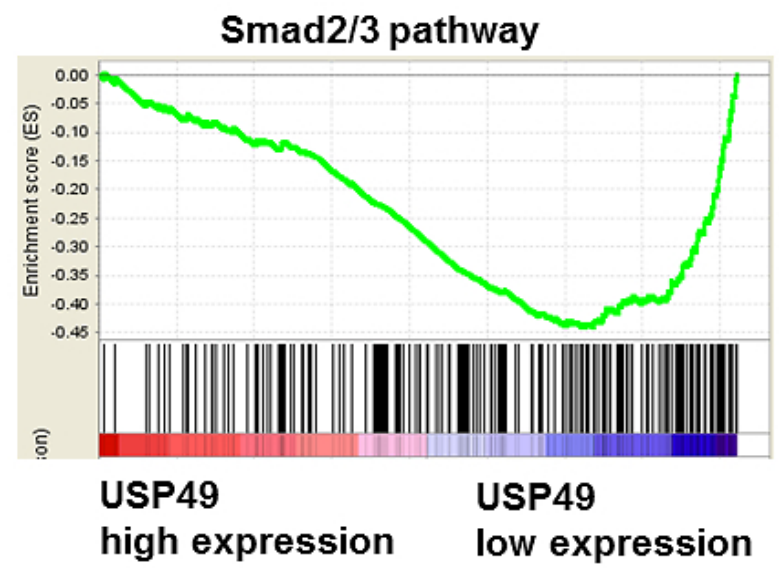



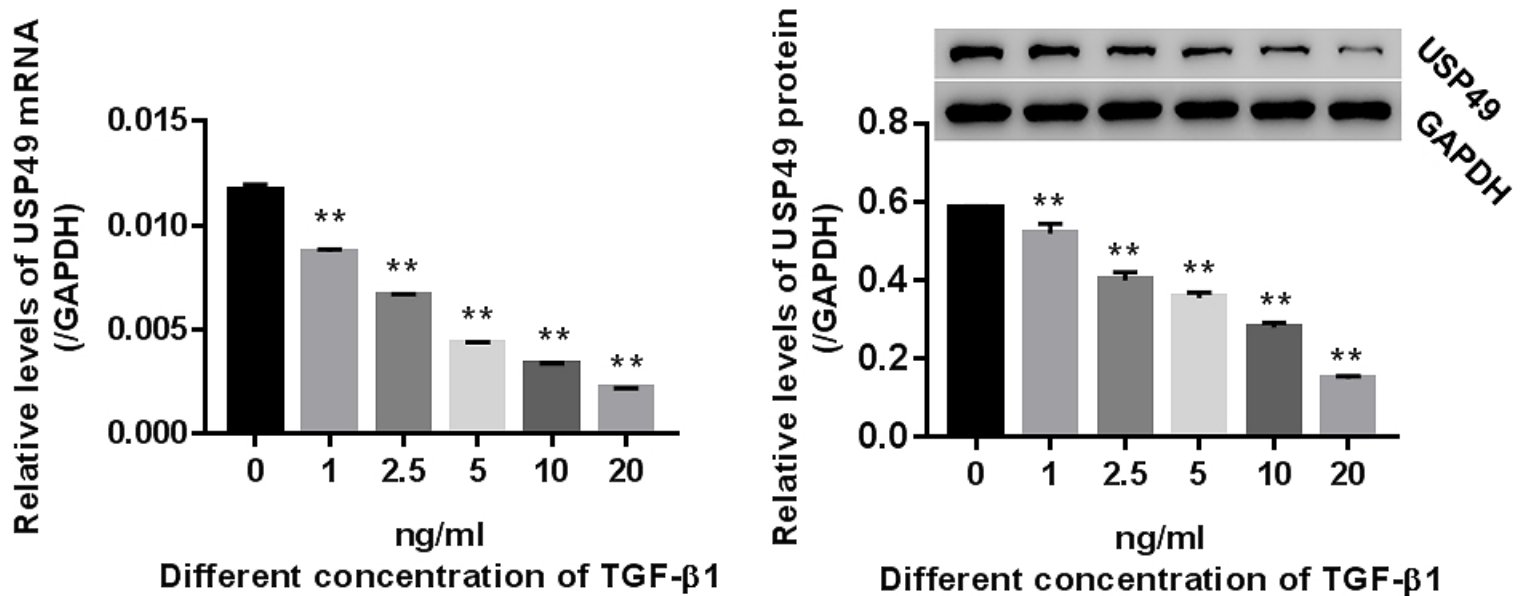


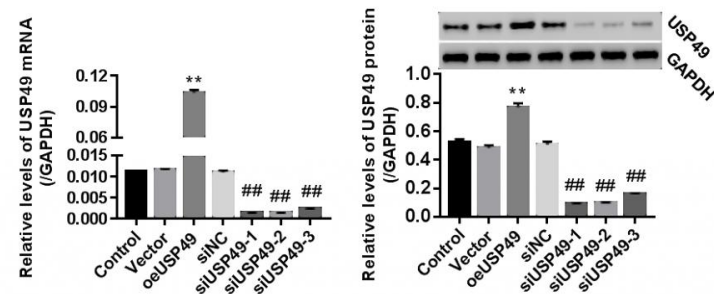

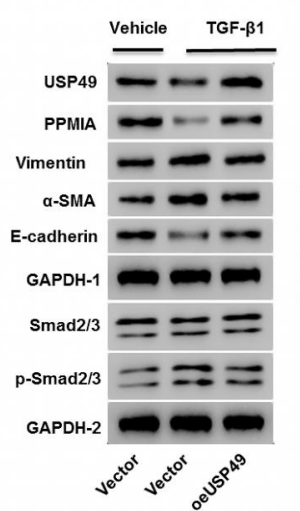

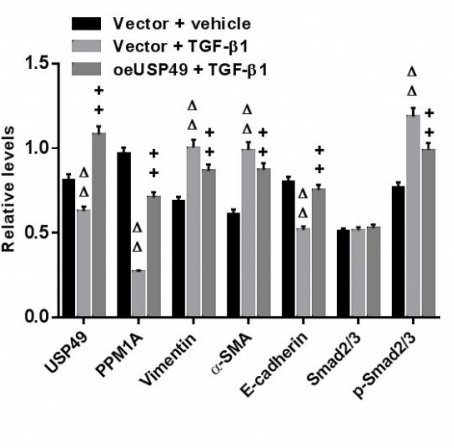

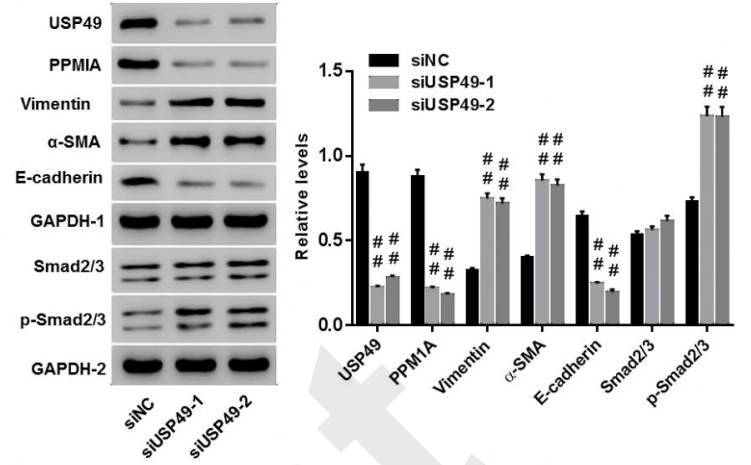

Figure 3-revised 
A

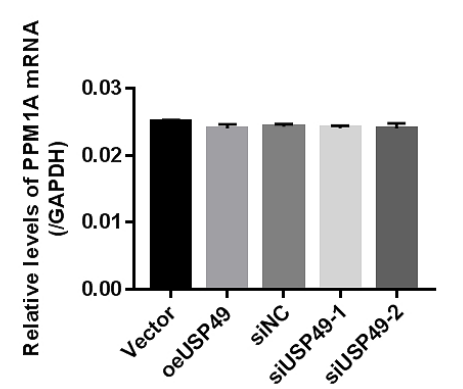

C

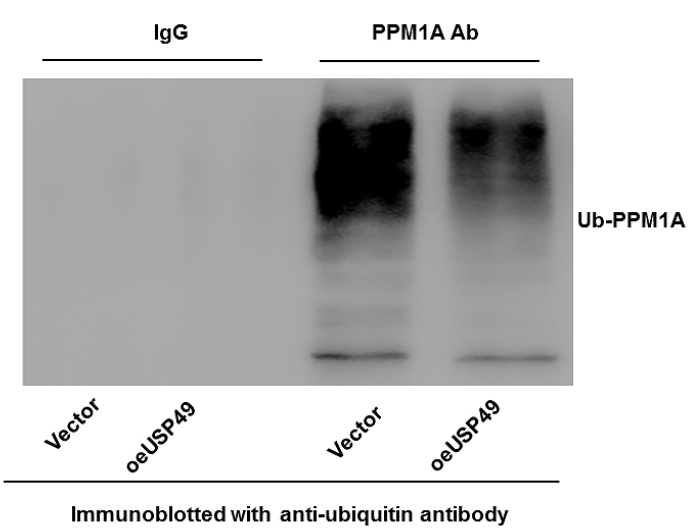

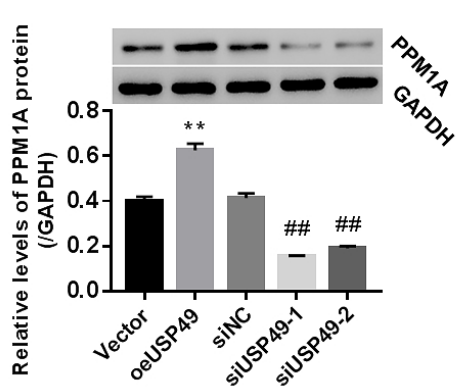

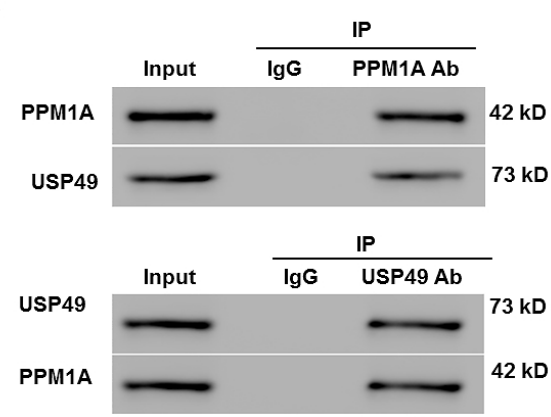

D

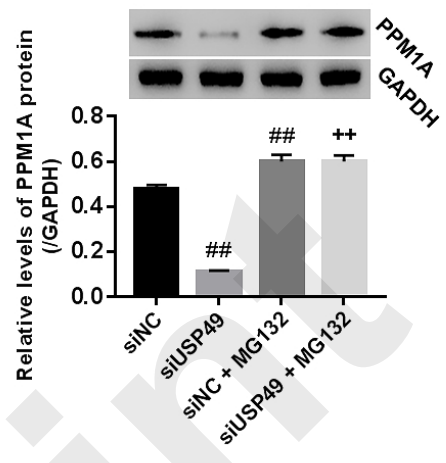



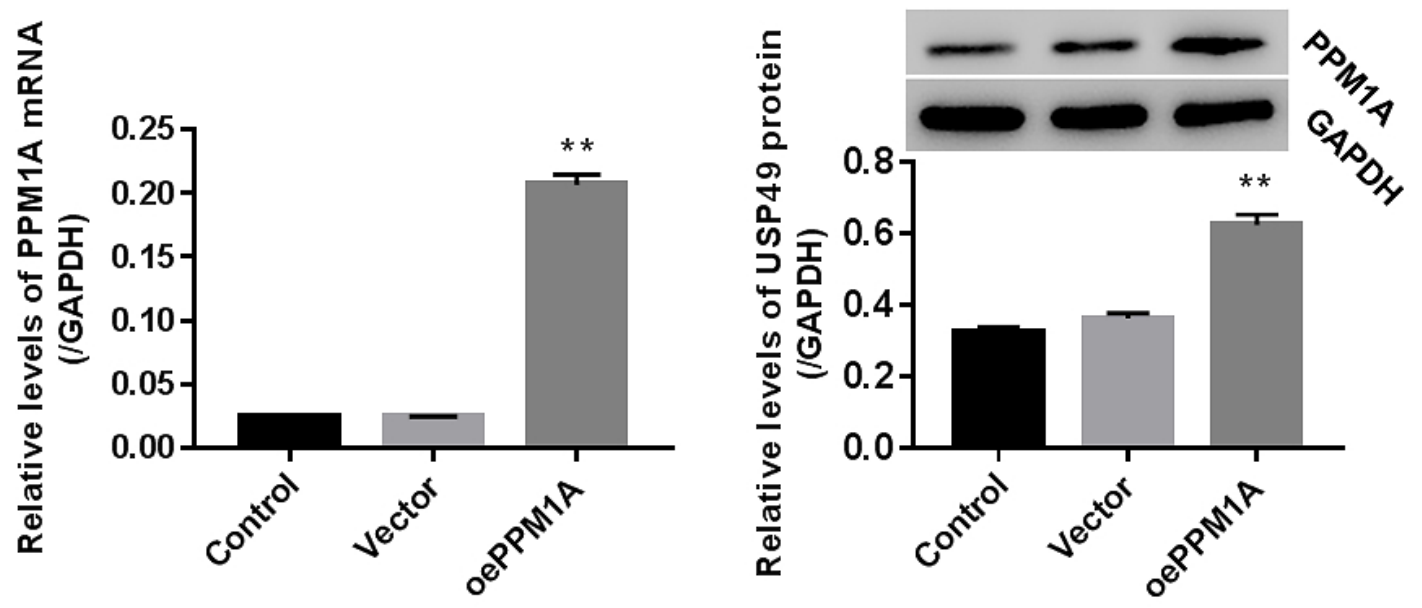

B

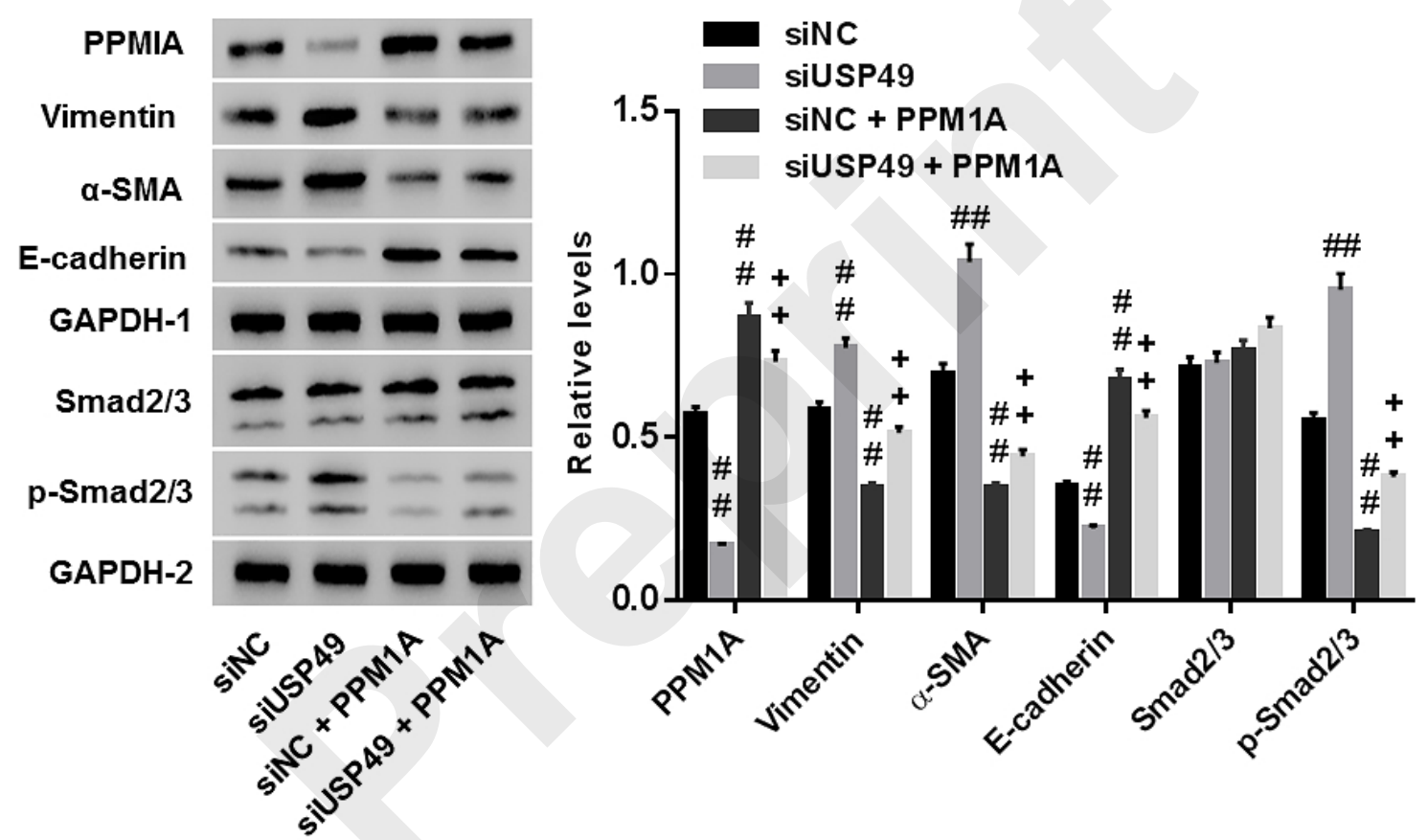


A

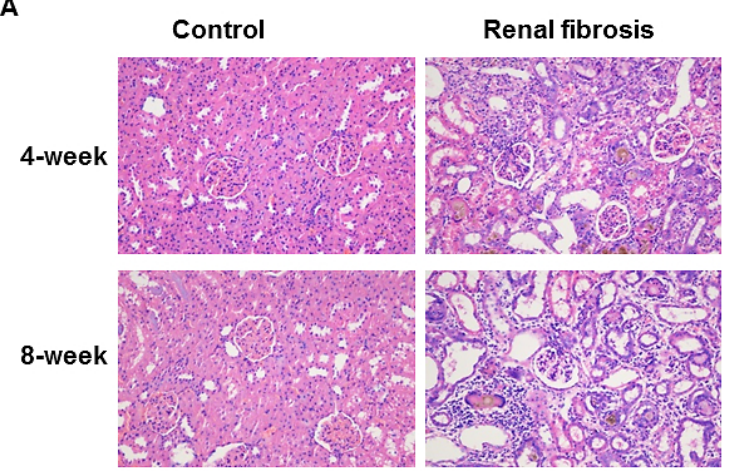

B

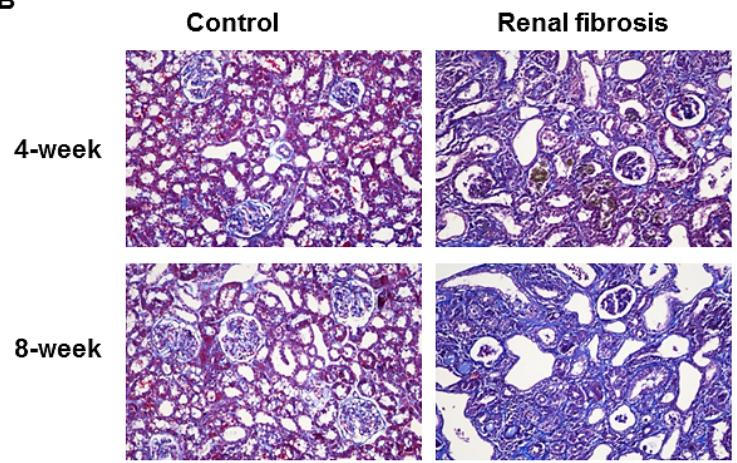

C

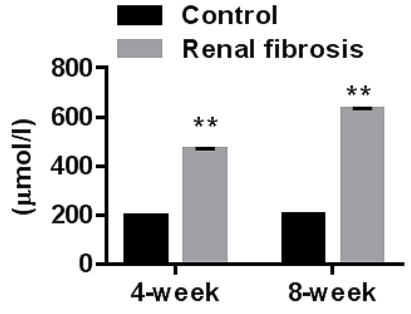

Creatinine in serum

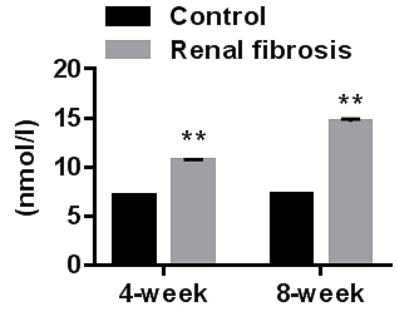

Urea nitrogen in serum

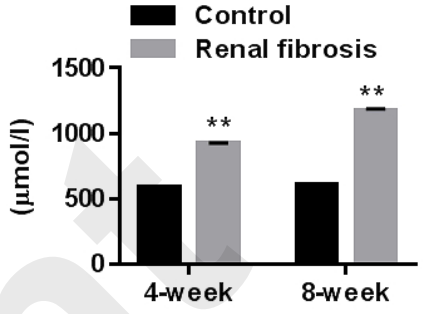

Urinary protein in urine

D
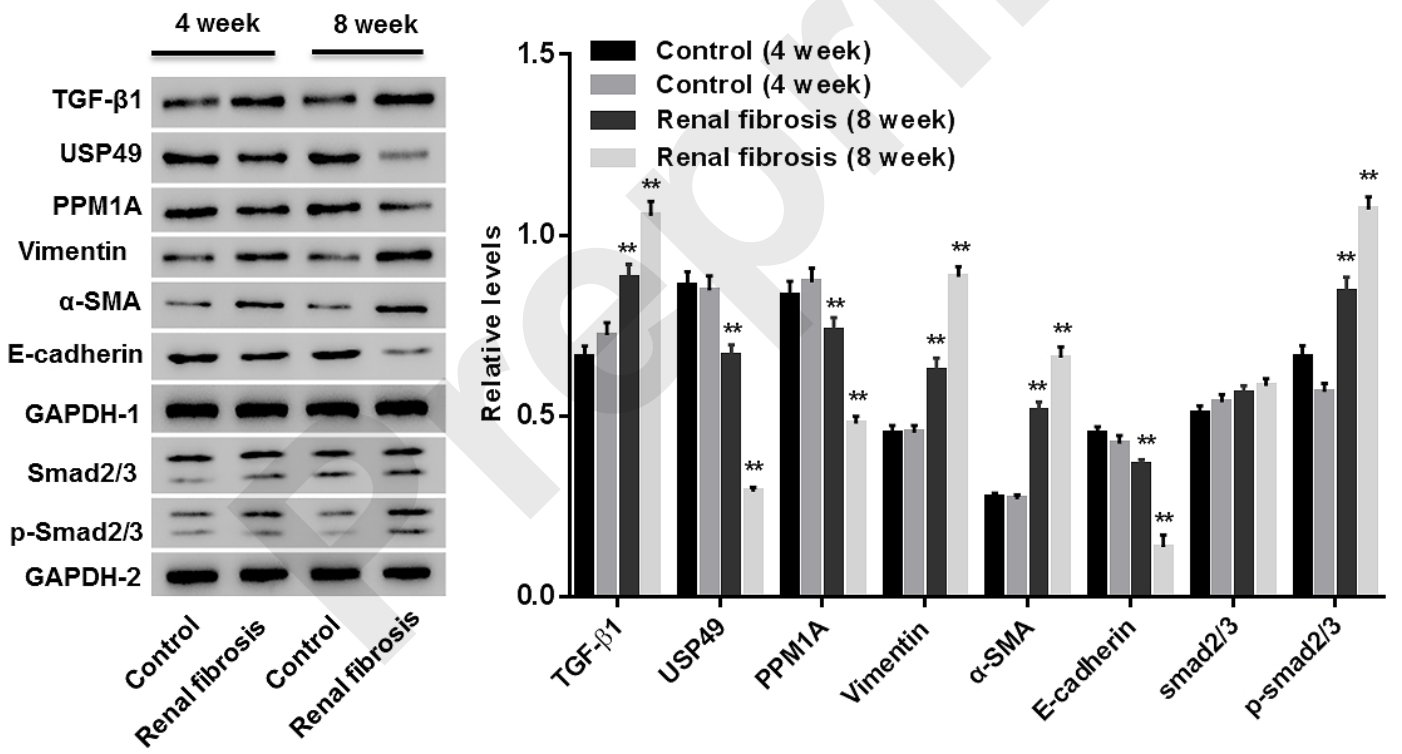